\title{
Model of Formation of Cognitive Strategy in Children of 7-11 Years, Specifically Taught Memorisation Techniques
}

\author{
Georgy Andreevich Sapozhnikov
}

Centre of Education 1679, Russian Federation, 125239, Moscow, Novopetrovskaya street, $1 \mathrm{~A}$

\author{
Doi:10.5901/mjss.2015.v6n5s1p159
}

\section{Abstract}

The article aims to analyse the results of the implementation of extra-curriculum classes of mnemonics, logical reasoning, and analytical reading in primary school. The results of children who went through the course are compared against the results of those who did not. The results show a considerably more serious competency level of children who took the classes. Reasons of success of them are scrutinized. A system is described in detail, examples of exercises and games are provided. Author offers a list of techniques for teaching. Structure of the course is included. Criteria for results evaluation is offered. A cognitive model of transformation of visual memory into logical reasoning is proposed. Author describes the psychological principles that underlie mnemonics and reading strategies of children and puts forward a cognitive model of dimensional memorisation as well as dimensional comparison. The author seeks to explain the qualitative difference between innovative approach of teaching children to memorise specifically and the old approach, by which this skill is not taught and accepted as natural. The article lends credence to the theory of strong connection between cognition, memory and intellect.

Keywords: mnemonics, primary school, analytical reading, logical reasoning, memory, cognitive science

\section{Introduciton}

Mnemonics is a set of techniques aimed at acquiring a skill of effective memorising.

Advanced visual and associative thinking contributes to the most reliable memorising. With the help of mnemonics it is possible to memorise textual, digital, and symbolic information. For the visual thinking and mnemonic memorising to form as a skill, it was decided to implement an extra-curriculum lesson of mnemonics and analytical reading in primary school.

For the realisation of this program an agreement was achieved to set up a test ground in Centre of Education 1679 in Moscow.

Mnemonics can be helpful by itself. However, the aim of this particular course was to teach mnemonics as a fundament for analytical reading and logical reasoning.

Hypothesis 1: Before adapting the course for the primary school, the author had been teaching mnemonics and analytical writing to people of different age using the methodology of Nikita Viktorovich Petrov, PhD. This methodology set the grounds for the course in the primary school. Every age group had its specificities, but in general they did not manage to form a skill, but rather obtained the knowledge of mnemonic techniques. This was the reason for attempting starting a course in younger ages, when skills get formed more successfully.

Hypothesis 2: Formation of integral visualised summary of text helps to find the links in otherwise unconnected pieces of information, and substitute linear search with a dimensional analysis, thus improving cognitive processes.

Innovative approach of this methodology lies in teaching children to memorise specifically. Normally, the methods of memorising are not studied in school, it is taken for granted that children know how to do it properly. On the other hand, introducing a separate class for learning the method of cognition will form a cross-subject basis for more effective learning process.

Super-voluntary memorising - a term of V.A. Kozarenko, an author of mnemonics system "Giordano". This memorising differs from the ordinary one in that means systematised memorisation in a given limited time period.

Recollection - voluntary retrieval of information from the brain. Differs from "remembering" in speed of the process.

Database - a system of associative links in the memory. It allows to voluntary remember the information memorised with the help of key images.

Reading by words - the process of reading, by which every word is perceived as an integral object and is appreciated without prior pronunciation. 
Abductive reasoning - logical reasoning that goes from an observation to a hypothesis.

\section{Methodology}

\subsection{Scientific basis and fundamental principles of mnemonics and analytical reading}

Let us apply a theory of memory mechanisms by V.A. Kozarenko. One can get more information from the book (6). What is relevant in the context is a thesis taken from this book. It states that a person does not memorise information, but rather generates it in response to the stimuli from the outside. Kozarenko maintains that not the separate items but rather the links between them are the units of memory. He offers a simple mental experiment. Seeing a word "breakfast" everyone imagines something, and it is very unlikely that all the people on Earth will have the same image come to their mind. This word will start a signal that leads to, for example, "a plate of flakes", of "a sandwich", or "a cup of coffee", as everyone has a different idea of a "breakfast". However, those images only appeared when the word had been taken in. This is, they were generated in response to the stimulus. For such stimuli, which narrow down the list of things recalled to only the relevant ones, Kozarenko establishes a term "spatial frequency filter".

Using the mute articulation (or sub-vocalization) is not recommended in mnemonics as some fixed speech constructions (e.g. idioms) can contain false connections, and this can lead to mistakes in memorisation. Thinking words impedes memorising. For all the reasons mentioned above the process of memorising will be reduced to formation of visual connections and a time standard will be established for creating one connection. It was calculated from the experiments and equals to 6 seconds - a period, necessary for a brain, which is not experienced with mnemonic memorisation, for creating a stable visual ties. This allows to optimize the process of memorising and introduce a standard for successful memorising. With practice, this period can be reduced, but cannot not theoretically go down below 0,14 seconds - a time, needed for an electronic signal to go down the nerve.

Theory of generative grammar of Noam Chomsky leads credence to the theory of Kozarenko. Basing on the former one George Miller, a psycholinguist, noted that an average adult is capable of understanding more than $10^{20}$ sentences, which can hardly be explained if we assume an unconscious accumulation of associations (14). This, in turn, means that associations allow to reduce the amount of efforts needed for memorisation in the process of cognition. A theory of Robert Lockhart (13) says that the level of processing of information influences the memorising. This processing comes to organising ties. For example, if we need to learn what an internal combustion engine is, we can define it as "the main part of a vehicle" or "a main part of a vehicle which applies the thermal power of combustion" of "a main part of a vehicle, which applies the thermal power of combustion, independently invented by Benz, Daimler, and Maybach". The latter has much more chances to be memorised. The other side is the speed of memory vanishing after contact with information, and the same law claims it is the slower the more processed the information was.

Concerning acquiring skills, one can recall the experiments of Paul Kolers. People under experiment were learning to read a reflected text (4). The result showed that the skill remained without regular practice for around a year. This allows us to assume that the skill of mnemonic memorising and analytical reading, once soundly obtained, will persist for at least the same period. In fact, they should remain forever, as, in contrast to the one of Kolers' experiment team members, will unavoidably be practiced regularly.

\subsection{Attention and Concentration}

Lack of attention in students can become a major problem. Students distract themselves by not only purposefully doing something unrelated to the task, but also by unconscious movements, like fidgeting, dandling pencil, or wiggling a foot. If no one pays attention to this problem in childhood, this can cause major trouble to an individual once he or she becomes an adult, when they appear to be unable to concentrate on a single task.

If we speak of theory, distraction interferes with the operation of spatial frequencies filters. A man aimed at recovering associations with, for example, a word "breakfast", other thoughts, say, related to the "dinner", hamper the process of remembering. If we consider memorising, poor attention can create false association, as the brain will have to process several images. This theory is coherent with ideas of Donald Broadbent. He presented them in his book "Perception and Communication" (3). The works (7), (16), and (17) represents the idea of visual search and makes it apparent that a distracted person loses memorising capability. Appendix A has several exercises to improve concentration. 


\subsection{Reading by Syllables and Reading by Words}

One of the principal goals set in the beginning of the course was to increase the speed of reading. Rapid reading is based on several methods to achieve this. To those belong:

1. Suppressing the "mute speech", forming the habit of discerning words as images and reading texts through visual perception channel, bypassing audio channel.

2. Practicing active reading, by which the brain filters excessive words. For example, fusional languages, of which Russian language is a good example, prepositions and conjunctions, as well as other function words, are coherent with changes in word endings. If one misses them in reading, one can increase the speed of reading dramatically.

3. Understanding the main idea of a sentence in contrast to additional information, structural relationships between them.

4. Studying the rules of writing different types of texts (social and political journalism, scientific articles, critical essays, fiction) with an aim to develop skills of searching reading.

5. Widening the visual angle and training recognition of symbols that lie away from focal point of an eye.

However, children under the age of 10 still go through a process of vocal apparatus formation. This means, suppressing the "mute speech" is not desirable. Moreover, reading plays a major role in the learning the mother tongue. Because of this, omitting function words can have negative effect. These are the reasons the methodology for primary school does not apply the two first methods.

Majority of adults develop a skill of reading by words (rather than by syllables) independently. They can train a skill of reading further to become capable of reading by entire sentences and even, in certain situations, by paragraphs. On the other hand, the children in primary school have word-reading as their first objective. As a result, the program included it as a specific goal of reading classes in 2-4 forms. In certain situations children developed this skill by the end of a course, but the majority learned to confidently read by syllables. If a child receives individual lessons, he or she can learn to read rapidly even earlier. The program in this case, however, included only one group lesson a week. The initial level of every child was too different. It seemed much more reasonable to form a steady fundament in every child than to acquire individual exceptional results.

One can train word-reading through exercises on fast word discernment. The words start from short conjunctions and gradually grow. Methodology of O. I. Krupenchuk (11) includes many of those. Through practice with such exercises, children compile a "cognitive bank of word images" and increase the speed of reading.

\subsection{Techiques}

A one-year course is split into following blocks:

1. Fundamental principles and methods of mnemonics. Learning to connect images and form databases.

2. Forming of multi-level databases with a voluntary access to any information in them.

3. Reading by words (by syllables for the first form).

4. Separating the main information from the collateral. The idea of a pronouncement.

5. Developing the skill of memorising separate pieces of text and their mental comparison for the production of an integral idea. Graphical epitomizing. Informational graphics. Mind maps.

6. Studying of consistent and inconsistent narration. "What came first - what came after?". Making up the first and the last part of the story basing on the middle part.

7. Studying the structure of different types of text.

8. Logics. Sufficiency and insufficiency of premises. Consistent and inconsistent reasoning. Grounded and ungrounded conclusions. Induction, deduction, and traduction.

9. Figurative sense and metaphors. Parables. Literary tropes.

10. Comparing new information against the one learned before.

Additionally, the course includes different tasks for developing psychological stability, creativity, imagination, attention, brain hemisphere synchronization, and brain multitasking.

In this article, we see these stages as milestones. Basing on them, we will make an effort to propose a systematic guide for further practical utilization. This is the first declared purpose of this work.

The second purpose is to offer scientific explanation and propose a cognitive model of brain operation, on which this methodology is based. 
1. Mnemonics applies mental connection of images. If something that one wants to memorize is a material object, one needs to imagine it and connect with something that is already known. The latter should also take a form of an image. If something that one wants to memorize is a thing of a higher abstract level, one needs to come up with an image that can serve a good representation of this abstract thing, something that is strongly associated with it.

Following are some of the methods that utilize this principle on which the entire mnemonics resides (20).

"Links" - a sequence of images that represent the words for memorising is linked in pairs. For example, we need to memorize four words: dipper - bear - chocolate - construction site. We imagine two neighbouring images, i.e. dipper and bear - and connect them in a non-standard way: a bear plays with a dipper, puts its nose inside, and cannot remove it.

The combination should be kept in mind for 6 seconds. It is important to keep exactly two images in a certain instance.

Lets pay attention to the word combination "construction site". It is a more abstract concept, and it implies an aggregate of several items. For this reason a student needs to make up an image that represents a construction site in his perception himself. The association personally devised have higher memorising potential.

"Magnifier" - the fundamental principle is similar to the one of the previous method, but in this situation the second object is imagined as a part of the first. For example, the fur of the bear becomes a multitude of tiny chocolate bars upon zooming in.

Cicero Method - the principal method, by which the things to be memorised are associated with the elements of a familiar list, for example, items in a house.

The so-called "memory route", combined with a rule for moving from one object to another (say, clockwise), gives an empty "database", in which the required information can be "written".

Henri Bergson studied Cicero method in his work "Two Forms of Memory" (2). Here Bergson divides the memory into two categories: biographical factual memory and memory for purposeful memorising. This is a widely acclaimed model in the cognition science.

Hermann Ebbinghaus (8) has work on the edge effect. He produced the curves of forgetting, and they showed that the first and the last elements of the list have stronger positions for memorising. This problem disappears by the application of Cicero method, as each element comes separately.

2. Cicero method is the key not only to memorising, but also to quick retrieving information from memory. It is possible to construct separate routes for different subjects, and thus make search faster. This neutralizes the problem with linear memorising, when losing one intermediate element can destroy the whole picture. It also improves analysis, as images can be compared more easily.

3. Analytical reading starts after familiarization with mnemonics techniques. The following methods entered the program:

a. Searching reading (looking for an answer for a particular question, suitable for reading academic literature). Ann Trisman, a student of Broadent, created a Feature Integration Theory. It predicts that it is easier to find an object with one distinctive feature, rather than several. This is why it is crucial to teach children to form proper retrieval requests and read the text purposefully rather than passively.

b. Analytical reading (skimming the paragraphs for the main idea, studying the structure of the text, and reading the text specifically to memorize).

c. Widening the visual angle using Shulte tables.

d. Anticipation of the text basing on the emotional context, common sense and the general knowledge.

e. Practicing reading with "curtain". A piece of paper goes down the page with a constant speed thus pushing to read quicker.

f. Adjustment method. The text is read 4 times in a scanning mode, and a self-assessment of comprehension is produced after each attempt.

4. One needs to learn to separate relevant information from the irrelevant. Combined with the methods of mnemonics it settles the fundament for the rapid reading. To train this skill the program included topiccomment analysis of a sentence and the following exercises:

a. Reduction of a sentence to the main parts of sentence, i.e. subject and verb, in successive steps.

b. Extending the sentence in successive steps by adding one word at a time.

c. Composing a story out of sentence with only subjects and verbs.

d. Exercises for practicing intonation, comparing the methods of putting an emphasis in writing and in 


\section{speaking.}

Superposition of topic-comment and mnemonics gave the children an opportunity to memorise the information in small parts rather than in bulk.

5. It is vital that children exercise retelling. For example, such a game can be a good exercise. Children get split into teams, and each of the three members receives a unique text for a limited time. They have to read each his/her text, and retell to the others. The time allocated is calculated in such a manner that it is impossible for each team member to read the whole text him or herself, but, on the other hand, it is necessary that everyone knows the content of each text as the questions require comparison and synthesis of information from different pieces. The children are advised to read and retell, taking out the unnecessary and sharing the most important.

6. Two exercises can be offered to practice understanding the structure of text and consistency of the text:

a. Children get the opening part of the story and they have to produce a coherent story.

b. Children get a final part of the story and they have to make up the beginning.

7. The texts studied were split in three types: fiction, publicism, and popular science. At a start, the children were plainly reading separate texts. On the latter stages (5-6 weeks after start), when they had already gathered a portfolio of texts, children discussed the texts and their peculiarities, basing on which they can apply different strategies of reading. The following points are crucial:

a. The tone and the language of narration (official, friendly, emotional, didactic).

b. Schemas (the style that allows the use of particular schemas, which type of text requires a verbatim understanding, and which requires to understand the expressive means of language).

c. The sequence of narration (general to particular, deduction; particular to generalization, induction; For example, the popular science articles typically have introductory part with problem overview and a conclusive part with summary, which states the same that was already said; in the purely scientific ones, for example, the ones from encyclopaedia, however, the information only comes up once, the events are put in a chronological order; fiction normally follows ideological coherence, but not always chronological).

d. Authors position (fiction - metaphorical and dispersed over the whole text; critical article and publicism as a rule, present in the last paragraph; purely scientific text - not present).

e. Structure of a separate paragraph (publicism - the first sentence of each paragraph represents the main idea of this paragraph, the last sentence summarises the paragraph; scientific text - initial sentence gives idea of the theme of the paragraph, but the last one does not repeat anything; fiction - has no particular structure and based mainly on the idea).

\section{Results}

When attempting to memorise something completely new by methods of multiple repetition, a person produces onedimensional connections between two elements, thus forming a chain. The links in this chain can be, for example, sentences from a textbook. When this same person needs to recall, he has to go through all the same elements again. This can be not only time-consuming, but can altogether fail if one of essential links misses. Moreover, lacking the crosslinks, that same person might miss even the apparent connections. In "On Pedagogical Principles of Mathematics" M.V. Potockiy describes a situation, when a student made a supposition that a ball on a sloping panel will roll up, arguing that "in physics and mathematics it's all the other way round". V. Uspenskiy uses this situation in (18) to demonstrate what a detached cognitive model of the world an ordinary schoolchild can have. This happens because of linear memorisation.

What mnemonics offers instead is not a chain, but a network, where each element has multiple connections. A. Jost in (9) invented a differential law of memorising:

$$
\frac{d x}{d T}=m(\lambda-x)
$$

Which has a solution

$x_{T}=\lambda-\left(\lambda-x_{0}\right) e^{-m T}$

$[\mathrm{X}]$ - the strength of memorisation, $[\mathrm{k}]$ and [lambda] - constants, $[\mathrm{T}]$ - time or a number of attempts of memorising. This means that with each next repeating the increase in quality of memorising becomes exponentially smaller. Instead of repeating, the best strategy would be establishing mental ties and retelling. In the same time, forming properly organised mental databases should help to recall faster and compare the uncoordinated facts, which in turn, helps to improve logical thinking.

To prove this hypothesis an experiment was carried out. The children were offered similar tasks on inductive, 
deductive, and abductive reasoning in the beginning and the end of the course (Appendix $C$ includes the typical tasks). Completing 4 and more tasks is deemed successful. Table 1 provides information on both test (varying overall amount ensues from the dropping out of some students):

Table 1. Results of introductory test and final test of logical reasoning of children who went through the course

\begin{tabular}{|l|c|c|c|}
\hline & 2 form & 3 form & 4 form \\
\hline Introductory test & $3 / 23$ & $5 / 25$ & $15 / 38$ \\
\hline Final test & $14 / 18$ & $15 / 20$ & $28 / 30$ \\
\hline
\end{tabular}

On the other hand, the same final test offered to the ones who have not attended the course on mnemonics and analytical reading are offered in Table 2

Table 2. Results of test of logical reasoning of children who did not go through the course

\begin{tabular}{|l|c|c|c|}
\hline & $2^{\text {nd }}$ form & $3^{\text {rd }}$ form & $4^{\text {th }}$ form \\
\hline Final test & $4 / 40$ & $10 / 54$ & $10 / 53$ \\
\hline
\end{tabular}

This lets us make a supposition that learning to turn information into images plays a role in development of imagination, creative thinking, and an ability to find hidden connections. The following model of thinking seems credible: a child reads and memorises the task with the help of visualisation. His mental picture has three crates and one apple, which should be in one of them. The first and the second crates are removed from this picture as the boy fails to find an apple in them. Only one crate remains. The correct conclusion becomes apparent.

Appendix B has some tasks for development of metaphorical thinking.

Another type of task offered was a collection of three short stories with a set of questions (Appendix D). The most interesting observation was that almost no one managed to answer the questions, which required comparing the information from the stories under different titles.

To check a hypothesis that children were not used to using the external knowledge the next class of the same age was offered the text with all the headings removed. The success rate was almost $85 \%$. This experiment was carried out with the age group of 7-8, 8-9, and 9-10 years, and the results were identical. Only children of 10-11 years of age seemed to have eventually developed the skill of cross-text analysis independently.

The final test included the tasks on cross-comparison. The results are in Table 3.

Table 3. Results of Cross-comparison tests

\begin{tabular}{|l|c|c|c|c|}
\hline & $1^{\text {st }}$ forms & $2^{\text {nd }}$ forms & $3^{\text {rd }}$ forms & $4^{\text {th }}$ forms \\
\hline Results of cross-comparison tests & $54 / 63$ & $15 / 18$ & $18 / 20$ & $26 / 30$ \\
\hline
\end{tabular}

This lets us to suggest that children needs a special inducement to learn to integrate all their knowledge into one picture of the world.

\section{Discussion}

From the observations and the experiments already known to the psychology it is possible to propose a model for understanding the process of learning and new information acquisition of children, who learn logic through analytical reading.

P. Johnson-Laird (12) claims that while making logical operations a person forms a mental model. He came up with an idea that some terms can have a form of images, while other, which belong to a higher level of abstraction, can be formed without images. However, the experiments mentioned above let us believe that mental conversion of logical operands increases the speed of thinking and cognitive processes.

It is known that recollection of facts from personal biography is connected with activation of pre-frontal areas of the brain, while recollection of general knowledge (semantic memory) leads to activation of left temporal and left frontal areas of cortex (5). It is also known, that imagining an object activates the same areas of the brain as perception (1). This means that using mnemonic techniques, for example, the Cicero method, activates right frontal zone, while mental 
modelling activates left temporal and left frontal areas of cortex. The main assumption of the theory of mental modelling is the truth principle: a person is inclined to reducing the load of the operational memory by the means of constructing the mental models, which represent explicitly only that what is true and do not represent that what is false (19).

The so-called "Navon superletters" (big letters written with small ones) show that when the big letter coincides with the small letters the answers come quickly and easily. However, when they did not match, focusing on the small ones (tuning for the details) lead to more problems. This lets us assume that seeing the big picture interferes with seeing the details. The more recent researches concretize these findings. In work (10) it was proven that occipital left and right brain play different role in adjustment. The left lobe focuses on the details, while the right focuses on the global picture. When we apply this to the operations of detailed deductive or inductive reasoning used in our course, the occipital left brain is activated, while when children make conclusions, the occipital right brain activates.

\section{Conclusions}

The short-term results of the course are adoption of the terminology and methodology of mnemonics, visual thinking, and analytical reading. However, these are the results that were present in all age groups. On the other hand, the results, specific for these age groups, were concentrate around improvement of logical thinking and formation of a learning cognitive strategy that normally takes years (like new knowledge integration, reading by words, or deductive and inductive thinking). This strategy formation is the principal aim of studying at school according to Pligin (15).

The long-term results of implementing the course in the primary school can only be evaluated after 5 years and more. By this moment the children will reach the age, which gave a start to the whole idea of teaching mnemonics in the early age: the inability of students to transform knowledge of methods into skill.

The implementation of the program had certain drawbacks. First, one hour of lessons per week is very little. In fact, the training should take at least 6 hours per week for a skill to form. Thus, a lot was left for homework, and this is not always reliable. The future courses need to take this into consideration. Secondly, before start of the course the author was misinformed about the level of reading skill of children, and was told that all the children can read. This turned out to be false, and a big part of the program had to be changed in very short terms. This, in turn, caused a high level of rigidity of the program. As yet, it adapts very poorly to different levels of children. The syllabus and materials need further development to become more flexible.

The author will be happy if the methodology arouses interest. The author is happy to discuss the possible advance in techniques and ways of implementation, as well as to study the possible criticism.

\section{References}

Baars, B. (2007). Cognition, brain, and consciousness: Introduction to cognitive neuroscience. Amsterdam: Academic.

Bergson, H., \& Paul, N. (1912). Matter and memory,. London: G. Allen \&;.

Broadbent, D. (1958). Perception and communication. New York: Pergamon Press.

Cermak, L. (1979). Levels of processing in human memory. Hillsdale, N.J.: Lawrence Erlbaum Associates ;.

Conway, M. (2002). Levels of processing 30 years on: Special issue of memory. Hove: Psychology.

Derryberry, D. \& Reed, M. A. (1998) Anxiety and attentional focusing: Trait, state and hemispheric influences, Personality and Individual Difference, 25, 746-761

Dodd, M. (n.d.). The Influence of Attention, Learning, and Motivation on Visual Search.

Ebbinghaus, H. (1986). Human memory and cognitive capabilities: Mechanisms and performances : Symposium in memoriam Hermann Ebbinghaus 1885, Berlin Humboldt University 1985. Amsterdam: North-Holland ;.

Jost, A. (1897). Die Assosiationsfestigkeit in ihrer Abkängigkeit Von der Verteilung der Wiederholungen Von Adolf Jost. Hamburg: Voss. Kozarenko V.A. (2007) Handbook of Mnemonics, "Giordano" system.

Krupenchuk, O., \& Iferova, O. (2011). Teach Me to Read: Methodology of Step-by-Step teaching of Reading: . Saint-Petersburg: Litera. Johnson-Laird, P. (1999) Deductive Reasoning, Annual Review of Psychology, vol. 50, 109-135

Lockhart, R.\& Craik (1990). F. I. M. Levels of processing: A retrospective commentary on a framework of memory research. Canadian Journal of Psychology, 44, 87-112.

Miller, G., \& Laird, P. (1976). Language and perception. Cambridge, Mass.: Belknap Press of Harvard University Press.

Pligin, A. (2007). Cognitive Strategies of Schoolchildren. Moscow: Profit Style.

Sternberg, R. (1977). Intelligence, information processing, and analogical reasoning: The componential analysis of human abilities. Hillsdale, N.J.: Lawrence Erlbaum Associates;.

Tsai, Y. (2012). Effects of working memory capacity on workload and task performance.

Works of Non-Mathematics. (2012). Moscow: Mathematical sketches.

Velichkovskiy B. M. (2006) Cognitive Science: Fundamental of Cognitive Psychology. M: ACADEMA, 218 
Yates, F. (1966). The art of memory,. Chicago: University of Chicago Press.

Appendix A

Games for developing the psychological stability, creativity, imagination, concentration, and lobe synchronization.

1. Imagine a particular place and try to hear the sounds. Name as many sounds as possible.

2. Try to hold five matches between the corresponding fingers of different hands, i.e. one match between the thumbs, one between the forefingers and so on.

3. Keep focused on the second hand of the clock for 20 seconds while reading a small poem by heart. When the mind distracts, start all over.

4. Write a dictation with a non-master hand.

5. "A fly": draw a $5 \times 5$ grid on the desk and put a fly into one of the cells. The children close their eyes and the teacher tell out loud the dislocations of the fly. For example, "three cels up". At a certain moment the fly escapes the grid and the children must say when it happens.

Appendix B

Games and exercises for development of appreciation of metaphors and schemas

1. Provide antonyms for particular words.

2. Provide synonyms for particular words.

3. Provide analogy for a particular word or situation, compare it to something.

4. Provide word combination, in which a particular word is used with positive attitude, and then the combination, in which this same word is used with negative connotation (for example, "strong like an elephant" or "clumsy like an elephant").

5. Make up a proverb for a particular situation.

6. Make up an anti-proverb, which advices to go exactly the opposite to some known proverbs.

7. Make up a legend to explain some phenomenon (for example, why does the forks typically have four teeth?)

8. Make up a story one sentence at a time.

Appendix C

\section{Inductive and deductive reasoning}

Winnie-the-Pooh wanted honey. Only Rabbit and Piglet could treat him with some. On the other hand, Rabbit did not favour Winnie after his last visit.

\section{What conclusion can Winnie make?}

A lot of people think that ice skating is both art and sport. Mary wants to take up a hobby which will be both beautiful and athletic.

What can we advise Mary?

It is know that one of three crates has an apple in it. A boy put his hand in two of them and found no apples.

What is the conclusion a boy can make if he thinks thoroughly?

\section{Abductive reasoning}

Victor, Shaun, and Arthur organized a chess competition. Victor thinks: "I am quite a poor player, so Arthur will definitely win".

What is the assumption that underlies the conclusion of Victor?

Peter wants to become a good boxer. He bought himself mufflers. "Now I am definitely going to become a good boxer" - Peter thinks.

What is the principal assumption of Peter?

My granny and I can prepare a flower bed in half a day. I said: "Let me prepare the flower bed myself. I will then be able to prepare it in one day".

What is the conclusion basing on? 
The paper is made out of wood. If we recycle the used paper, we can use it all over. This means, we can cut down a finite number of trees and have enough paper forever.

\section{What is assumed in the argument?}

\section{Appendix D}

\section{SEPTEMBER (S)}

The cold mist cover hollows and river valleys in the mornings. By the midday, the sun manages to warm the air and it becomes almost as in summer. September is the most beautiful month of autumn: a wind blows and the forest drops down a waterfall of golden and red leaves. The cover the ground like a colourful carpet. A solemn charm resides in the woods. The silence of birds is particularly noticeable. Only titmouse might give a whistle at times.

WINTER CAME $($ WC $)$

A cold wind started blowing from the north, and snowflakes are falling from the skies. They whirl in the air and fall down, and you cannot pick the nicest one. Here is a flower with six petals, here is a six-point star, and this is a thinnest plate with six edges. The more quiet the weather is, the more beautiful are the snowflakes falling down. Strong wind brakes their rays and edges and the white flowers turn into dust. But when the cold is frugal, the snowflakes roll into solid white balls, and we call it sleet. The fields are all white, and no one is around.

\section{NOVEMBER'S HERE $(\mathrm{NH})$}

The days of the late autumn are dim and short. The long nights are impenetrably dark. The skies are low and overcast. "The November breaths an autumn chill..." One sees no leaves on the trees. Only to wolf the late autumn and winter are no wonder. The beast wanders the lonely fields freely. In Novembers the wolves do not live in the big woods, they run in packs from thickets to grassy swamps, to low forests, to ravines and closer to villages. The short November day decreases quickly. It's getting darker. A smoky veil of twilight floats, and the precincts grow cloudy. In the evenings, crows and jackdaws reach for the village, and the magpies - for the forests. In the mornings it is the other the way round: crows fly to the forest and the magpie go to the village. This is a good guiding line for a lost hunter. Use the flight of birds to get out of the forest.
1. What is called "a flower with six petals"? (WC)

2. What do we call a sleet? (WC)

3. What is a "solemn charm"?

4. What do winter and November have in common?

5. If the weather is bad in winter, how will the snowflakes look like?

6. What is the difference between the November and the September?

7. Both September and winter have something beautiful. What is it in September and what is it in winter?

8. Put a "+" if the information stated can be inferred from the text, and "-" if it cannot. If you put "+" find a quote for a proof:

a. How can you get out of the forest if you get lost in September?

b. How many points does a snowflake have?

c. When is the day the shortest?

d. What birds do not fly away in November?

e. Do wolves live in packs in September?

f. What time of the day is it cold in September? 